\title{
SUBMAXIMAL LABORATORY ANAEROBIC TEST TO DETERMINE RHYTHMIC GYMNASTS' FITNESS LEVEL
}

Maria Gateva

National Sports Academy "Vassil Levski", Sofia, Bulgaria

\begin{abstract}
In sports such as gymnastics it is difficult to monitor the fitness and endurance level of the athletes. In rhythmic gymnastics it is proven that the competitive program is in anaerobic regime of work. Some authors state that there is no contribution by the aerobic energy system in gymnastics. Very few attempts have been undertaken to create specific anaerobic tests in the different gymnastics disciplines. That's why the aim of this study was to create a laboratory test to measure the fitness level (specific endurance) of the rhythmic gymnasts competing at national and international level.

Twelve high level gymnasts aged 15.7 ( \pm 2.1$)$ were involved in this study. Two tests were performed in the laboratory - $\mathrm{VO}_{2}$ max and submaximal 2-min test (author's test) and one field test-competitive routine was carried out. Two min min submaximal treadmill test with constant load (95\% of the maximal test) requires a 2-min run on a treadmill with a constant speed of $8,4 \mathrm{~km} / \mathrm{h}$ and individually set up incline of $95 \%$ of the maximum for each athlete. The incline is fixed on the basis of: reached HR and incline during the maximal test; extrapolation of the incline as $100 \%$ from the theoretical maximal pulse and calculation of the $95 \%$ of the incline for each gymnast.

Rhythmic gymnastics routine clearly showed better results in the competitive period and is an indicator for tapering into peak performance. We received some contradictory information as regards the laboratory results. Heart rate during the whole load on the treadmill test was lower (significantly proven). This means that in the next stage of the investigation, the experiment needs to be done with the same duration, but we should increase the load to $100 \%$.
\end{abstract}

Key words: rhythmic gymnastics, laboratory test, submaximal load, fitness level

\section{INTRODUCTION}

In sports such as gymnastics it is difficult to monitor the fitness and endurance level of the athletes. The $\mathrm{VO}_{2}$ max test which is a gold standard for measuring the aerobic endurance in many sports is not applicable in disciplines where the anaerobic system is the energy supplier. The anaerobic regime of work has been proven to be considerably activated in rhythmic gymnastics competitive program (Gateva \& Andonov, 2006; Gateva, 2008; Manos, Grigore, Popescu, 2012; Batista, 2018), but there are not enough investigations in this field. We believe the comparison with other gymnastics disciplines' kinematic structure of the movements and duration of the routines is adequate and inevitable because they are closest to rhythmic gymnastics. In artistic gymnastics, the estimated aerobic energy supply in floor routine is no more than $20-30 \%$ while the main energy supplier is anaerobic glycolysis (Jemni et al. 2011; Marina, \& Rodríguez, 2014). Powers \& Howley (2018) state that there is no contribution by the aerobic energy system in gymnastics. We completely disagree with the statement that floor routine in artistic gymnas- 
tics and routine in rhythmic gymnastics are predominately dependent on aerobic metabolism (Armstrong \& Willem, 2017).

Very few attempts have been undertaken to create specific anaerobic tests to measure the fitness level of the athletes in the different gymnastics disciplines (Alves et al., 2015; Heller et al., 1998; Gateva, 2019). Heller et al. (1998) suggests that a field test of 30-s repetitive jumping performance may be used as a simplified measure of anaerobic capacity, because of its close relationship to the standard laboratory 30 -s Wingate test.

In laboratory conditions Force-Velocity and Wingate tests which measure the power output are not ideal to determine the specific fitness level in rhythmic gymnastics despite the fact that authors are using them in artistic gymnastics (Jemni et al., 2006). The Runningbased Anaerobic Sprint Test (RAST) is similar to the Wingate Anaerobic and the tree-minute step test uses comparison of personal results obtained from different testing (Mackenzie, 2005). Douda et al. (2006) suggest that rated perceived exertion scale (RPE) could be more efficient than heart rate in estimating the $\%$ of $\mathrm{VO}_{2}$ max in gymnasts of different competitive levels (especially for higher level gymnasts) in a training program. The variables of heart rate and subjective rate of perceived exertion are significant to control the effects of training on the performance of individual rhythmic gymnasts (Fernández-Villarino, et al., 2018).

Based on the physiological indicators of a competitive routine the aim of this study was to create a laboratory test to measure the fitness level (specific endurance) of the rhythmic gymnasts competing at national and international level.

\section{METHODS}

\section{Subjects}

Twelve high level gymnasts (some in the
Bulgarian National team) aged $15.7( \pm 2.1)$ were involved in this study. The competitors were category "Elite" from Levski Triaditsa sport club in Sofia, training 30 to $35 \mathrm{~h}$ per week. The testing was done twice - first in the preparatory period and then in the competitive period. Two tests were performed in the laboratory $-\mathrm{VO}_{2}$ max and submaximal 2-min test and one field test - competitive routine was carried out. Parental permission and informed consent were obtained from all participants. The study protocol was conducted in agreement with the principles stated in the Declaration of Helsinki for human studies (WMJ, 2008) and in compliance with the ethical code of the National Sports Academy (2019).

\section{Instruments}

Heart rate was recorded with POLAR RCX3. The recording started at rest and continued until $10 \mathrm{~min}$ post exercise (recovery period). Blood samples were taken from the fingertip to measure blood lactate with the use of an Accutrend Plus Roche. Lactate was taken before and on the $3^{\text {rd }}, 5^{\text {th }}$ and the $9^{\text {th }}$ min during the recovery period (Weinstein et al. 1998). Based on those two indicators it is possible to compare the load during all the tests.

For the laboratory testing COSMOS $\mathrm{h} / \mathrm{p}$ (Germany) treadmill was used. Gas exchange was monitored during and $10 \mathrm{~min}$ after the exercise (load). Breath by breath gas exchange was measured continuously with Oxicon Pro (Yeger, Germany), blood pressure and other associated metabolic and cardiovascular functions indicators were recorded and will be presented in later publications.

\section{Study design}

Laboratory testing (on treadmill)

The first task was to assess the fitness level of the gymnasts with standard testing as $\mathrm{VO}_{2}$ max and based on the results to design a 
test with a load close in duration and intensity to the rhythmic gymnastics routine. This new test needed to provoke work at $95 \%$ of the reached maximum $\mathrm{HR}$ during the $\mathrm{VO}_{2}$ max test.

The 2 treadmill tests:

1. $\mathrm{VO}_{2} \max -\mathrm{a}$ modification of Balke Treadmill Protocol was done - Athlete (Franklin et al., 2000). Increase of the incline of the treadmill with $0.6^{\circ}$ (starting from $0^{\circ}$ ) on each $30 \mathrm{sec}$. The speed during the test was constant $-8.4 \mathrm{~km} / \mathrm{h}$. to determine the level of the load reached at the end of the test in order to calculate the submaximal load for the 2 min test. It was done only once - in the preparatory period.

2. 2-min Submaximal treadmill test with constant load (95\% of the maximal test) (authors'test) - The test requires a 2-min run on a treadmill with a constant speed of $8.4 \mathrm{~km} / \mathrm{h}$ and individually set up incline of $95 \%$ of the maximum for each athlete. The incline is fixed on the basis of:

- reached HR and incline during the maximal test;

- extrapolation of the incline as $100 \%$ from the theoretical maximal pulse and calculation of the $95 \%$ of the incline for each gymnast.

The 2-min submaximal laboratory test was performed 48 hours after the maximal one took place. The test was performed in the preparation and in the competitive period.

\section{Field testing:}

1. Competitive routine - individual rhythmic gymnastics competitive routine with average duration $1.30 \mathrm{~min}$. The field testing was done in the same week of the laboratory testing at the beginning of the training session in the preparation and in the com- petitive periods.

\section{Warm up}

The start of each of the laboratory tests was preceded by a three-minute light warm up. When testing in field the routine was accomplished at the beginning of the training sessions after a 30-min warm up, followed by a 10-min specific warm up prior to the routine. After completing each test, the subjects were asked to have a passive recovery period for 10 $\min$.

\section{Statistical analysis}

Descriptive statistics was used to characterize the physiological reaction during and after applying the tests and routines in rhythmic gymnastics.

Student's paired t-test was applied to find the differences in heart rate monitoring and blood lactate measuring between the two periods - preparatory \& competitive period. Student's independent t-test was used to compare the laboratory 2-min test with the load provoked by the rhythmic gymnastics' individual routine. The level of significance of $p \leq 0.05$ was adopted in all cases.

\section{RESULTS}

The $\mathrm{VO}_{2}$ max reached for this group was $50.0( \pm 4.2) \mathrm{ml} / \mathrm{min}^{-1} \mathrm{~kg}^{-1}$ and some individuals reached $57.0 \mathrm{ml} / \mathrm{min}^{-1} \mathrm{~kg}^{-1}$. Blood lactate didn't show any significant difference in preparatory period between the two testing - in lab and in field. Contrary to this, the heart rate from the beginning of the load to its end had a significant difference $(p \leq 0.01)$. Figure 5 clearly shows that the 2-min laboratory test had lower values and could not reach the target of the competitive routine. 


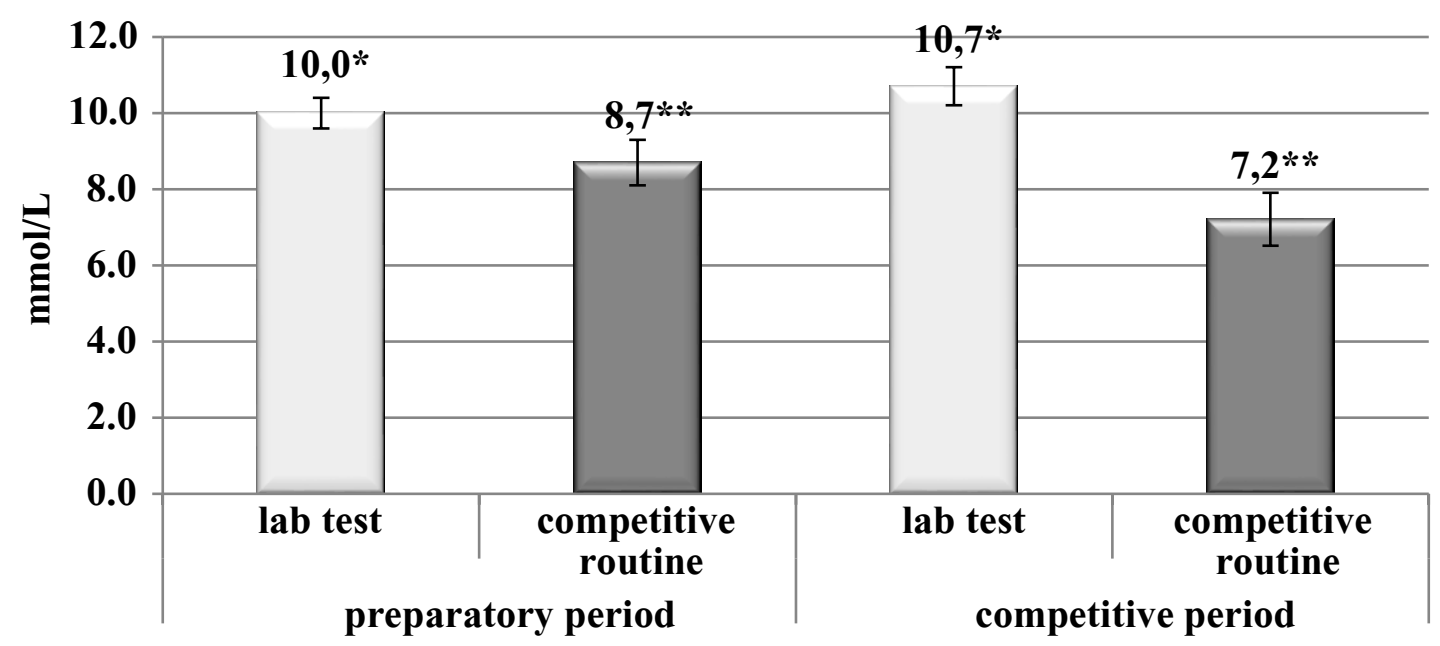

*difference between prep period to competitive in lab test

** difference between prep period to competitive in routine

Figure 1. Blood lactate values after submaximal lab test \& routine

In the competitive period the average val- ferences. ues of blood lactate had differences between the lab and field testing. In the laboratory test no improvement was observed in La and even the values were slightly higher but with level of significance $(p \leq 0.05)$. From the $30^{\text {th }}$ second of the loading onwards, the heart rate had differences $(p \leq 0.01)$. But at the end of the routine and the lab test the peak values showed no dif- $\quad(p \leq 0.05)$.

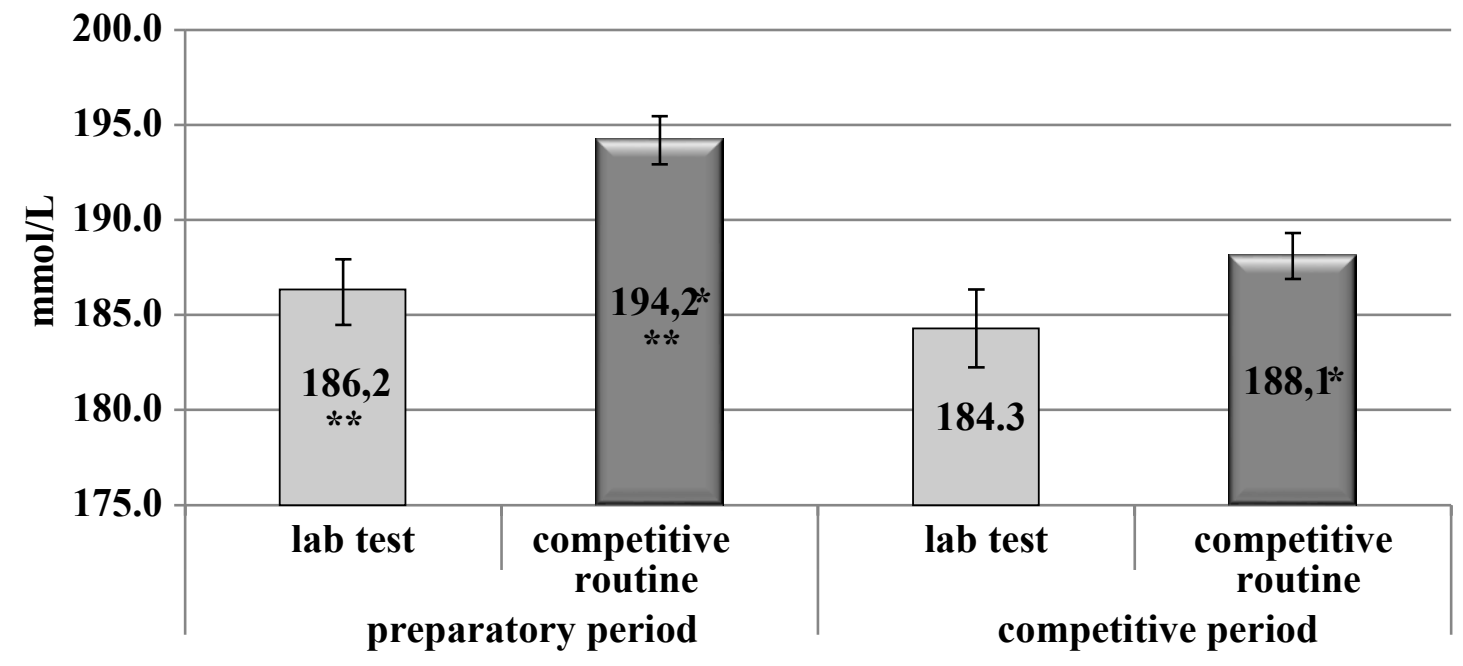

*difference between prep period to competitive in routine

** difference between lab test \& routine in prep period

Figure 2. Heart rate values after submaximal lab test \& routine 
Table 1. Descriptive statistics of the HR and concentration of the blood lactate after the load

\begin{tabular}{lcccccc}
\hline Test & $\begin{array}{c}\text { HR mean } \\
(\mathbf{S D}) \\
(\mathbf{b} / \mathbf{m i n})\end{array}$ & $\begin{array}{c}\text { HR min. } \\
(\mathbf{b} / \mathbf{m i n})\end{array}$ & $\begin{array}{c}\text { HR max. } \\
(\mathbf{b} / \mathbf{m i n})\end{array}$ & $\begin{array}{c}\text { La mean } \\
(\mathbf{S D}) \\
(\mathbf{m m o l} / \mathbf{L})\end{array}$ & $\begin{array}{c}\text { La min. } \\
(\mathbf{m m o l} / \mathbf{L})\end{array}$ & $\begin{array}{c}\text { La max. } \\
(\mathbf{m m o l} / \mathbf{L})\end{array}$ \\
\hline Laboratory test PP & $186.2( \pm 6.0)$ & 178 & 198 & $10.0( \pm 1.6)$ & 7.2 & 12.3 \\
Gymnastics routine PP & $194.2( \pm 4.4)$ & 187 & 200 & $8.7( \pm 2.3)$ & 5.7 & 12.8 \\
Laboratory testCP & $184.3( \pm 6.5)$ & 174 & 193 & $10.7( \pm 1.6)$ & 8.0 & 14.1 \\
Gymnastics routine CP & $188.1( \pm 4.0)$ & 181 & 194 & $7.2( \pm 2.2)$ & 5.0 & 11.7 \\
\hline
\end{tabular}

$P P$ - preparatory period; $C P$-competitive period

The measured indicators during and after to the preparatory period. From the $45^{\text {th }} \mathrm{sec}$ rhythmic gymnastics routine clearly showed ond of the routine onward up to its end on the that the gymnasts tapered into peak perfor- $90^{\text {th }}$ second the difference was also significant. mance in the competitive period. The blood There was a significant difference in the relactate values decreased significantly, and the covery from the end of the load to the $4^{\text {th }} \mathrm{min}$. HR values were lower with $5 \mathrm{bpm}$ compared

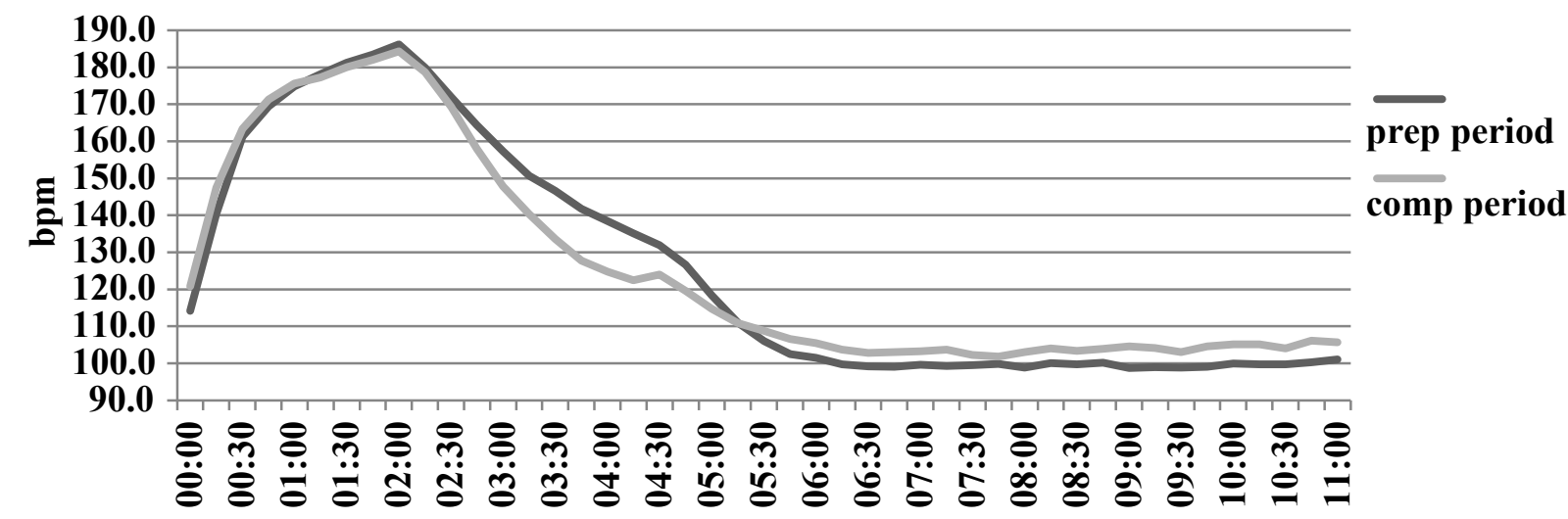

Figure 3. Heart rate during and after 2 min submaximal tresmill test in the preparatory \& competitive periods

Table 2. Heart rate dynamics during the testing (means $\pm S D$ )

\begin{tabular}{|c|c|c|c|c|}
\hline \multirow[b]{2}{*}{$t(\min )$} & \multicolumn{2}{|c|}{2 min laboratory test } & \multicolumn{2}{|c|}{ Gymnastics routine } \\
\hline & $\begin{array}{c}\text { Preparatory } \\
\text { period }\end{array}$ & $\begin{array}{c}\text { Competitive } \\
\text { period }\end{array}$ & $\begin{array}{l}\text { Preparatory } \\
\text { period }\end{array}$ & $\begin{array}{c}\text { Competitive } \\
\text { Period }\end{array}$ \\
\hline 00:00 & $113.8( \pm 17.6)$ & $120.8( \pm 17.5)$ & $147.5( \pm 11.0)$ & $132.9( \pm 17.6)$ \\
\hline 00:15 & $140.1( \pm 13.9)$ & $147.5( \pm 12.3)$ & $161.0( \pm 12.1)$ & $152.5( \pm 10.7) *$ \\
\hline 00:30 & $161.5( \pm 12.4)$ & $163.3( \pm 8.3)$ & $177.3( \pm 7.8)$ & $173.1( \pm 6.4)$ \\
\hline 00:45 & $169.5( \pm 10.4)$ & $171.3( \pm 7.3)$ & $186.2( \pm 5.3)$ & $180.5( \pm 5.3) *$ \\
\hline 01:00 & $174.9( \pm 9.1)$ & $175.6( \pm 5.6)$ & $189.9( \pm 4.9)$ & $184.5( \pm 4.0) *$ \\
\hline 01:15 & $178.1( \pm 7.9)$ & $177.2( \pm 5.8)$ & $191.6( \pm 4.4)$ & $186.1( \pm 4.1) *$ \\
\hline 01:30 & $181.2( \pm 6.9)$ & $180.0( \pm 5.8)$ & $194.2( \pm 4.4)$ & $188.1( \pm 4.0) * *$ \\
\hline 01:45 & $183.3( \pm 6.7)$ & $182.0( \pm 6.1)$ & & \\
\hline 02:00 & $186.2( \pm 6.1)$ & $184.3( \pm 6.5)$ & & \\
\hline \multicolumn{5}{|c|}{$* p<0.05$ significant difference between the periods } \\
\hline & innif & between the pe & & \\
\hline
\end{tabular}




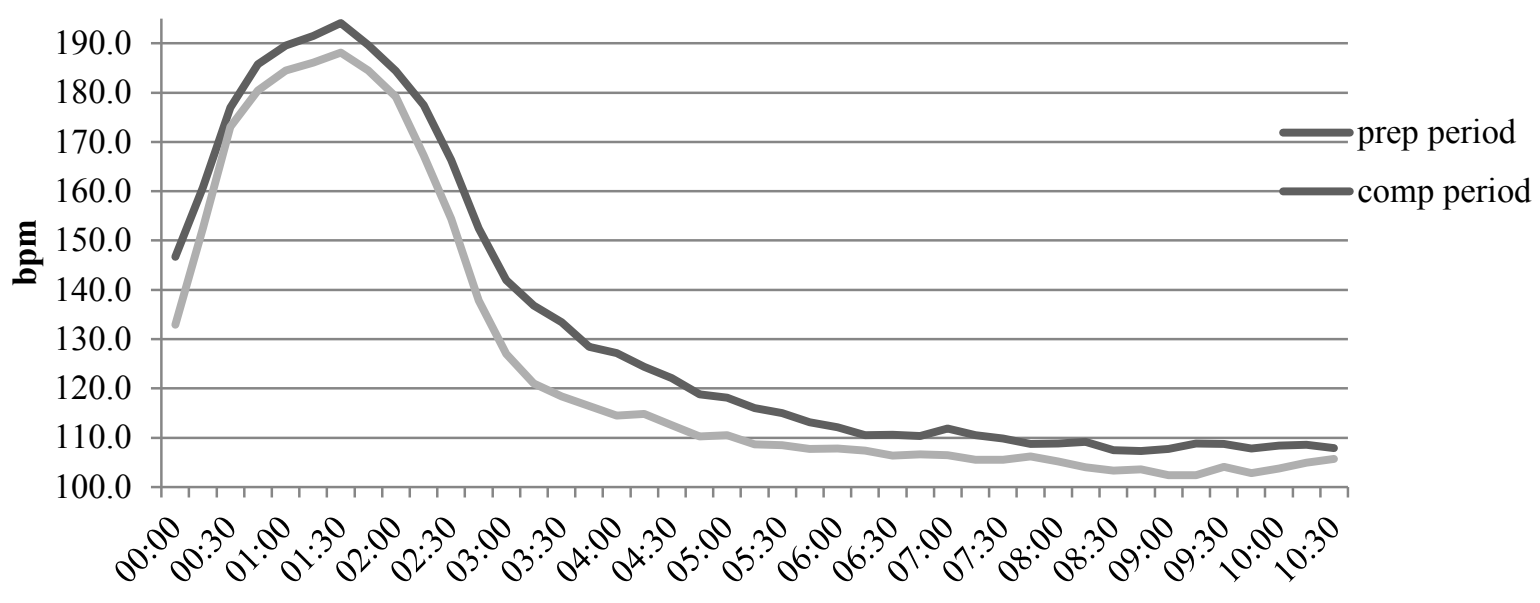

Figure 4. Heart rate during and after rhythmic gymnastics routine

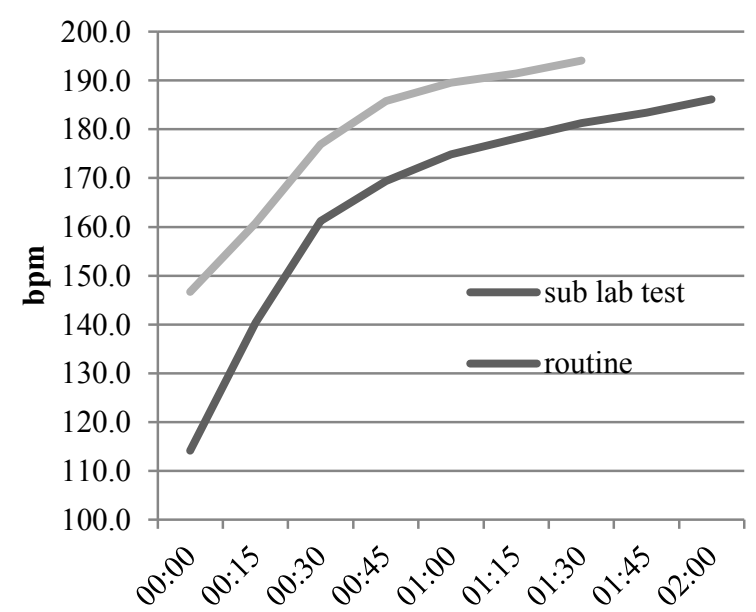

Figure 5. Heart rate in preparatory period

\section{DISCUSSION}

To discover if the applied new laboratory test is adequate and corresponds to the real load in rhythmic gymnastics (the competitive routine) we compared and analysed the obtained results from the heart rate and blood lactate in the two testing - in lab and in field. As we do not have another tool to measure the field physiological changes during the routine without influencing the execution of the gymnasts, the oxygen consumption and all related data from the laboratory testing will be part of another publication.

This study is part of a massive project in all gymnastics disciplines (Gateva et al., 2013)

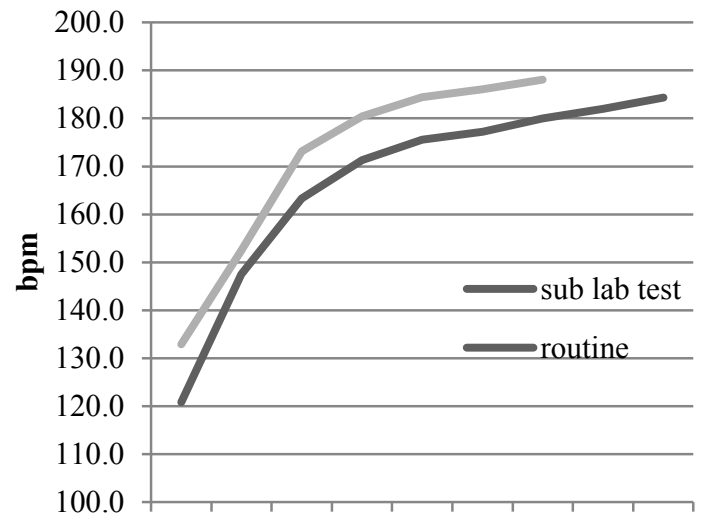

Figure 6. Heart rate in competitive period

to set up specific endurance tests (in lab and in field - 5 in total) for measuring gymnasts' fitness level.

In our study $\mathrm{VO}_{2} \max$ was completed in order to calculate the individual $95 \%$ for the 2-min submaximal test. Marina \& Rodríguez (2014) also made a comparison of the metabolic parameters recorded during the treadmill test and the competitive routines in women artistic gymnasts and found that floor routine reached $97 \%$ of the maximal values obtained during the incremental test. The choice of the protocol for our test - constant speed with load increase through increasing the incline of the treadmill was made considering the subjects' 
age (14-18). With the performance of 2-min submaximal treadmill test we had only several cases (5 gymnasts) who reached the theoretical maximal pulse and respective incline recorded during the $\mathrm{VO}_{2} \max$ test. For most of the subjects the greater incline was on their second (submaximal) test (it was extrapolated for their theoretical maximum). The choice of the 2-min submaximal test on a treadmill was based on:

$\checkmark$ Duration is comparable with the two gymnastics disciplines - individual and group (1.30 and 2.30 respectively) routine;

$\checkmark$ Intensity and effort should be close to the actual gymnastics routine $-95 \%$ of the maximum.

$\checkmark$ It is expected to measure the anaerobic and the beginning of aerobic capacity (same as gymnastics);

$\checkmark$ It is set up individually for each subject.

Routine is a control tool for the real load of the gymnastics discipline. Studying the physiological exertion (tension) in gymnasts' body during the execution of the competitive routine is necessary for a better understanding of the adaptation of the body's systems towards the intensity and the volume of the competitive impact (Gachev \& Djarova, 1992; Weltman, 1995; Grancharov, 1997; Gateva \& Andonov, 2006; Jemni et al., 2011). The higher intensity level in the training (moderate to very vigorous intensity exercises) corresponds to practising the gymnastics routines and parts of the routine (Batista et al., 2018).

What was clear from the received values from the rhythmic gymnastics routine is that it showed big improvement (significantly) and tapering into the peak performance period. We received contradictory information as regards the laboratory results. First of all, not all of the gymnasts managed to reach their maximal
$\mathrm{HR}$ in the $\mathrm{VO}_{2} \max$ test. Consequently, in the submaximal test some gymnasts had greater incline that the one reached in the incremental test. Observing the HR values in the preparatory period from the beginning to the end of the load we can see how 2-min submaximal test had lower impact on the body compared to rhythmic gymnastics routine. This will provoke another experiment in order to test the 2-min submaximal treadmill test to become maximal (goes up to gymnasts' maximum $100 \%$ ). Blood lactate was slightly higher in lab test but without any significant difference. In the competitive period the situation was controversial - blood lactate stayed above 10 $\mathrm{mmol} / \mathrm{l}$ (even increased its values) and the HR had not changed much from the preparatory period into the competitive one. Meanwhile the HR values during the gymnastics routine went lower (because of the adaptation) but still weren't higher than the laboratory results. In agreement with Thompsen et al. (2007), the best results in sport are obtained with the use of training protocols which are as specific as possible to the demands of the sport activity. The differences are possibly due to the type of measurement performed. It is not the same measuring in a laboratory conditions with nonspecific tests as in a real training situation. Because of the different kinematic structure of the running and gymnastics exercise we can explain the higher values in blood lactate. Also because of the adaptation, the trained muscles are able to cope with higher values of blood lactate (in competitive period) which can be produced by nonspecific exercise (running for gymnasts). HR during the recovery can be used as a tool to control and monitor athletes' current level of fitness and subsequent periodic testing can be used to evaluate the success of training programs. In some cases, time or distance covered would be the dependent variable measured rather than a direct measurement of 
power output to create sport specific test (Powers \& Howley, 2018). It appears in gymnastics we need greater effort in finding the best laboratory test. We should also try to standardise the effort in performing exercises so that field test be the most accurate - closest to the competitive programme.

\section{CONCLUSIONS}

In the attempt to find an adequate standardise laboratory test to measure the specific endurance in rhythmic gymnast we found several issues:

Heart rate during the whole load on the treadmill test was lower (significantly proved). In the next stage of the investigation the experiment needs to be done with the same duration of the test (2 min) but to increase the load to $100 \%$;

since a gymnast doesn't practice running the adaptation in this test will be difficult to observe as in the rhythmic routine the tapering into the peak performance period was very clear. The laboratory test can be used as a tool for indicating the taper since its recovery period values can show a quicker adaptation of the cardiovascular system.

\section{REFERENCES}

Alves, C., Borelli, M., Paineli, V., Azevedo, R., Borelli, C., Lancha Junior, A., Gualano, B., Artioli, G. (2015). Development of a Specific Anaerobic Field Test for Aerobic Gymnastics. PLoS One, DOI:10.1371/journal. pone.0123115 April 13.

Armstrong, N., Willem, M. (2017). Oxford textbook of children's sport and exercise medicine. Oxford University Press, Oxford.

Batista, A., Gomes, N., Garganta R., Avila-Carvalho, L. (2018).Training intensity of group in rhythmic gymnastics, Science, move- ment and health, Vol. 18 No1, pp. 17-24.

Code of ethics - National sports academy "Vasil Levski". (2019). Sofia. // Етичен кодекс на НСА „Васил Левски”. (2019). София.

Douda, H., Avloniti, A., Kasabalis, A., Smilios, I., Tokmakidis, S. (2006). Application of rating of perceived exertion and physiological responses to maximal effort in rhythmic gymnastics. International journal of applied sport science, Vol. 18 No 2, pp. 78-88.

Fernández-Villarino, M., Hernaiz-Sánchez, A., Sierra-Palmeiro, E., Bobo-Arce, M. (2018). Performance indicators in individual rhythmic gymnastics: correlations in competition, Journal of Human Sport and Exercise, Vol. 13 No3, pp. 487-493.

Franklin, B., Whaley, M., Howley, E. (2000). ACSM'S Guidelines for Exercise Testing and Prescription, Sixth Ed., American College of Sports Medicine, Lippincott Williams\& Wilkins, p. 219.

Gachev, E., Djarova, T. (1992). Biochemistry. Sofia, Nayka \& Izkystvo. // Гачев, Е., Джарова, Т. (1992). Биохимия. София, Наука и изкуство.

Gateva, M., Andonov, K. (2006). Characteristics of the effect of applied training loading in rhythmic gymnastics. Sport, stress, adaptation, proceeding of the international conference in Sofia, Bulgaria, Sport \& science, extra issue, pp. 63-69.

Gateva, M. (2008). Examination of the training loading in rhythmic gymnastics and perfecting the training methods. Doctoral dissertation. National Sports Academy, Sofia.

Gateva, M., Tarnichkova, M., Ivanov, N. (2013). Unified methodology for applying laboratory and field tests for different gymnastics disciplines. FIS Communications in Physical Education, Sport, and Recreation \& I International Scientific Conference. Nis, Book of proceeding, pp. 50-54.

Gateva, M. (2019). Modified field test for 
determining the specific endurance in rhythmic gymnastics. Journal of Applied Sports Sciences, Vol. 1, pp. 3-12.

Grancharov, N. (1997). Anaerobic threshold, the essence and applied value in sport. Sofia, NSA IPB. // Грънчаров, H. (1997). Анаеробния праг, същност $u$ приложна стойност в спорта. София, НСА ИПБ.

Heller, J., Tûma, Z., Dlouhá, R., Bunc, V., Nováková, N. (1998). Anaerobic capacity in elite male and female gymnasts. ActaUniversitatis $\mathrm{Ca}$ rolinae-Kinanthropologica, Vol. 34 No2.

Jemni, M., Sands,W., Friemel,F., Stone, M., Cooke, C.(2006). Any effect of gymnastics training on upper-body and lower-body aerobic and power components in national and international male gymnasts? The Journal of Strength and Conditioning Research, Vol. 20 No 4, pp. 899-907.

Jemni, M., W. Sands, J. Salmela, P. Holvoet, M. Gateva. (2011). The science of gymnastics. Routledge Taylor and Francis Group, London and New York.

Mackenzie, B. (2005). 101 Performance Evaluation Tests, Electric Word plc, London.

Manos, M., Grigore, V., Popescu, L. (2012). Study about the energy expenditure assessment in rhythmic gymnastics. Science, movement and health, Vol. XII, issue 2, pp. 170-175.

Marina, M., Rodríguez, F. (2014). Physiological demands of young women's competitive gymnastic routines, Biology of Sport, Vol. 31 No3, pp. 217-222.

Powers, S., Howley, E. (2018). Exercise physiology: theory and application to fitness and performance, tenth edition, McGraw-Hill Education, NY.

Thompsen, A., Kackley, T., Palumbo, M., Faigenbaum, A. (2007). Acute effects of different warm-up protocols with and without a weighted vest on jumping performance in athletic women. J Strength Cond Res. Vol. 21 No 1, pp. 52-56.

Weinstein Y, Bediz C, Dotan R, Falk B. (1998). Reliability of peak-lactate, heart rate, and plasma volume following the Wingate test. Med Sci Sports Exerc. Vol. 30, No 9, pp. 1456-60.

Weltman, A. (1995). The blood lactate response to exercise (monograph number 4) - Champaign, IL, Human kinetics.

World Medical Association, Declaration of Helsinki - Ethical Principles for Medical Research Involving Human Subjects. (2008). $W M J$, Vol. 54 No 4, pp. 122-125.

\section{Corresponding author:}

Maria Gateva

Department of Gymnastics National Sports Academy „Vassil Levski” 21 Acad. Stefan Mladenov Str. Sofia 1700, Bulgaria E-mail: maria.gateva@abv.bg 